\title{
Kein erhöhtes Risiko intrakanieller Blutungen unter niedrig dosierter ASS
}

Fragestellung: Ist das Risiko intrakanieller Blutungen unter der prophylaktischen Gabe von niedrig dosierter Acetylsalicylsäure (ASS) erhöht?

Hintergrund: Niedrig dosierte ASS ist die weltweit am häufigsten benutzte Substanz in der Sekundärprävention vaskulärer Ereignisse und bei Hochrisikopatienten auch in der Primärprävention. Die bisherige Datenlage zu der Frage, ob niedrig dosiertes Aspirin das Risiko für intrakranieller Blutungen erhöht, sind sehr widersprüchlich: So zeigten randomisierte Studien eher ein erhöhtes Risiko und Kohortenstudien kein erhöhtes Risiko.

Soriano LC, Gaist D, SorianoGabbaro M et al. Low-dose aspirin and risk of intracranial bleeds. An observational study in UK general practice. Neurology $2017 ; 89: 2280-7$
Patienten und Methodik: Die Autoren benutzten die Datenbasis der praktischen Ärzte in Großbritannien. Sie verglichen 199.079 Patienten, die mit $75 \mathrm{mg}$ bis $300 \mathrm{mg}$ ASS pro Tag behandelt wurden und im Alter zwischen 40 und 84 Jahren waren, mit einer gematchten Kohorte von Menschen, die keine ASS einnahmen. Die maximale Beobachtungszeit betrug 14 Jahre und der mediane Follow-up 5,4 Jahre. Das relative Risiko wurde für die Einnahme von ASS an den Tagen 0 bis 7 vor dem Eintritt der intrazerebralen Blutung berechnet.

Ergebnisse: In der Datenbank fanden sich 1.611 intrakranielle Blutungen, darunter 743 intrazerebrale Blutungen, 483 Subduralhämatome und 385 Subarachnoidalblutungen. Das relative Risiko für die Einnahme von ASS betrug 0,98 für alle intrakraniellen Blutungen, 0,98 für intrazerebrale Blutungen, 1,23 für Subduralhämatome und 0,77 für Subarachnoidalblutungen. Es ergab sich kein Zusammenhang zwischen Einnahmedauer, ASS-Dosis und dem Risiko.

Schlussfolgerungen: Die Einnahme in niedriger Dosis von ASS zur primären oder sekundären Prävention vaskulärer Ereignisse führt im klinischen Alltag nicht zu einem erhöhten Risiko intrakranieller Blutungen.

\section{Ein beruhigendes Ergebnis}

Diese Studie aus Großbritannien mit fast 200.000 Patienten und 200.000 Kontrollpersonen zeigt in beruhigender Weise, dass die Einnahme von niedrig dosierter ASS weder das Risiko intrazerebraler Blutungen noch das Risiko von Subduralhämatomen oder Subarachnoidalblutungen signifikant erhöht. Für Subarachnoidalblutungen fand sich sogar ein starker Trend für

eine protektive Wirkung. Die Ergebnisse dieser Studie sollen allerdings nicht darüber hinwegtäuschen, dass die niedrige Dosis von ASS insbesondere bei Personen in einem Alter über 75 Jahren signifikant das Risiko für das Auftreten oberer gastrointestinaler Blutungen und dadurch bedingter Todesfälle erhöht.

\section{Hormonale Kontrazeptiva und Risiko eines ischämischen Insults}

Die europäischen Fachgesellschaften EHF und ESC (European Headache Federation/European Society of Contraception and Reproductive Health) haben aktuelle Leitlinien zum Einsatz von hormonalen Kontrazeptiva bei Frauen mit Migräne vorgelegt. Generell ist bei Frauen, die unter einer Migräne mit Aura leiden, das Risiko ischämischer Schlaganfälle leicht erhöht. Das Risiko wird durch die Einnahme hormonaler Kontrazeptiva weiter erhöht. In den Leitlinien werden die bisher publizierten Daten zusammengefasst und es wird eine Therapieempfehlung abgegeben.

Insgesamt gesehen ist das Risiko eines Schlaganfalls bei Frauen, die eine Migräne haben, unter der Einnahme von hormonalen Kontrazeptiva nur gering. Kombinierte hormonale Kontrazeptiva gehen mit einem höheren Risiko einher als Monosubstanzen, insbesondere bei der Migräne mit Aura. Alles in allem liegt aber nur eine geringe Datenqualität bezüglich dieses klinischen Problems vor.

Hans-Christoph Diener, Essen

Sacco S et al. European Headache Federation and the European Society of Contraception and Reproductive Health. Hormonal contraceptives and risk of ischemic stroke in women with migraine: a consensus statement from the European Headache Federation (EHF) and the European Society of Contraception and Reproductive Health (ESC). J Headache Pain 2017; 18: 108 